\title{
NEARMesh: Network Environment Aware Routing in a Wireless Mesh Network for Emergency-Response
}

\author{
Tawfik Al-Hadhrami \\ School of Engineering and Computing \\ University of the West of Scotland, Paisley, \\ United Kingdom \\ tawfik.alhadhrami@uws.ac.uk
}

\author{
Faisal Saeed \\ Information Systems Department, \\ Faculty of Computing, Universiti Teknologi \\ Malaysia, Johor, Malaysia \\ faisalsaeed@utm.my
}

\begin{abstract}
Wireless Mesh Networks (WMNs) employ hybrid distributed mobile networks with instant deployment and capabilities Such as: self_healing, self_organization and self_configuration. These abilities make $\bar{W} M N$ s a likely technology for incident communication. An Incident Area Network (IAN) requires a reliable and efficient routing path in an environment, where infrastructure-based communications have been destroyed. Routing awareness plays a significant part in this situation to deliver dynamic disaster facilities. Though, most of the proposed aware routing schemes do not entirely exploit the characteristics of WMNs. In this article, we propose a network environment-aware routing scheme for emergency response (NEARMesh) in WMNs, which employs a network routing information map to select the optimized path, based on cooperative consideration of route awareness information. This scheme is carried out and verified in NCTNus simulator. Imitation outcomes clearly display that the suggested scheme can enhance the network performance by maintaining a high delivery ratio with low latency while reducing the energy ingesting by minimizing network expenses.
\end{abstract}

Keywords-Power Aware Algorithm; Routung Algorithm; WMN; Emergency-Response

\section{Introduction}

Reliable and efficiently-routed communication facilities are critical during emergency management. The aftermath of recent natural disasters (e.g., the Wenchuan earthquake in China and the Tsunami in Japan,) or terrorist attacks (e.g., the London attack and 9/11) has highlighted the fragilities and limitations of current infrastructure-based communication technologies [1]. In incident areas where pre-existing communication has been destroyed, emergency network communication (ENC) has to establish reliable and robust routes [2].

Wireless mesh networks (WMNs) [3] have acquired increasing momentum as a practical networking concept to restore communications instantly and cost-efficiently for emergency/disaster management. Despite the advances in the design and deployment of WMNs [4][5] a number of research questions remain unresolved. Network routing performance is a key performance objective of an emergency response network, in order to guarantee the network connectivity until the infrastructure is restored [5]. A number of routing-aware algorithms (e.g. for power level) [6] [7] have been proposed in the past years. However, these were mainly designed for generic mobile networking scenarios or highly static wireless networks. Moreover, they were incorporating power awareness only. In this article, a novel, integrated consideration routing awareness scheme for an emergency-response WMN is designed to achieve improved performance. Simulation results demonstrate that the proposed scheme significantly enhances incident area network operations in terms of routing performance (reducing routing overheads and end-to-end delay with increased packet delivery rate) and reducing power consumption in contrast to representative existing work and standard protocols. The article is organized as follows: the next section discusses related works, followed by a description of the NEARMesh scheme, the adopted OSPF protocol, the simulation results, and finally concluding remarks.

\section{Related Work}

Several routing algorithms using power consumption as the metric have been developed for WMNs in recent years [68]. These algorithms focus on searching for the optimal path with the minimum energy consumption. They are usually based on ad-hoc routing protocols and are designed for highly dynamic mobile networks. On the other hand, cooperationbased algorithms have also become popular recently [5]. Unlike the algorithms using the power metric, they incorporate the concept of cooperative communication to search for the shortest path from source to the destination.

The routing algorithms which only consider one aspect of the path features are not sufficient when dealing with the demanding requirements of emergency response scenarios. It is thus important to tackle the routing problems by considering both aspects: mesh node type and the power metric. In terms of the first aspect, an attempt has been made to establish the route from source to destination with reference to the mesh node types, such as route or client [3]. However, in this study the algorithm did not consider the power consumption of the routes and nodes, which is an essential factor for incident area networks (IAN). In [3] and an earlier study, [9], routing 
algorithms involving link qualities - ETX, ETT and WCETT were proposed. However, they neglected the importance of node status, e.g. live or dead, with regard to the routing performance.

In this paper, we propose the NEARMesh algorithm, which combines the metrics in terms of node efficiency (by including the neighbour's current power), link quality (by using ETX) and mesh node type. The proposed algorithm improves the performance of routing algorithms for IAN and addresses the gaps from the previous literature.

\section{The NEARMesh Scheme}

We consider a wireless mesh network consisting of a large number of mobile mesh routers (MR) and mesh clients (MC), as shown in Figure 1. Such a network model is an abstracted version of the WMNs deployed in incident areas, which support the communications for power suppliers, police officers, ambulances, operators and emergency agencies and for other emergency communication and management.

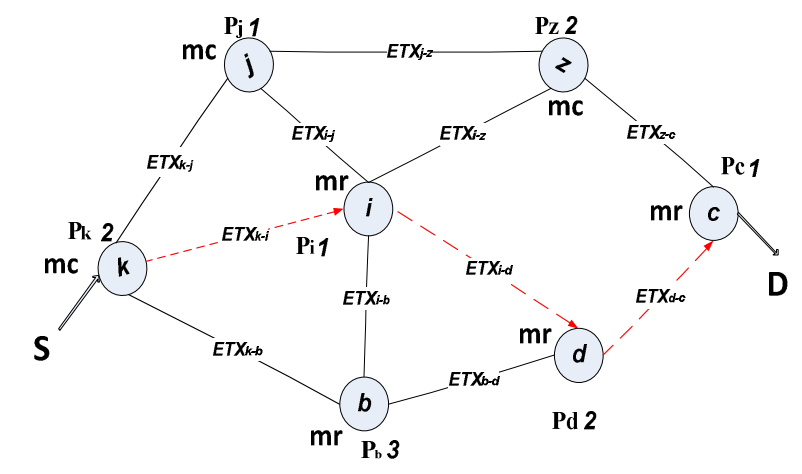

$--\mathrm{H}^{-} \rightarrow--\mathrm{H}^{2} \cdot \rightarrow--\mathrm{H}^{3} \rightarrow$

Figure 1 Rescue team Wireless Mesh Network Model

TABLE 1: THE ABBREVIATIONS USED IN THE ALGORITHM PSEUDOCODE

\begin{tabular}{|l|c|}
\hline \multicolumn{1}{|c|}{ Term } & Abbreviation \\
\hline PL & Initial Power \\
\hline Received Bit Power & PRx \\
\hline Transmit Bit Power & PTx \\
\hline Power Other Operations & POther \\
\hline Packet Power & PP \\
\hline Node ID & Nid \\
\hline Packet Size & S \\
\hline $\mathrm{n}$ & $\begin{array}{c}\text { Number of trials for successful recep. } \\
\text { m }\end{array}$ \\
\hline Current Power & transmission $_{\mathrm{t}+\Delta \mathrm{t}}$ \\
\hline Initial Power & $\mathrm{CP}_{\mathrm{t}}$ \\
\hline
\end{tabular}

\section{NEARMesh Scheme}

Send HL Signal to collect Neighbour Information

If $C P \leq L_{-}$Threshold Set $P L I=3$

else if $L_{-}$Threshold $<C P<H_{-}$Threshold Set $P L I=2$ end if

else if $C P \geq H_{-}$Threshold Set $P L I=1$

end if

Update Power Database Description(Source)

End if

If NodeType $==$ Mesh Router then

++ Mesh Router Count(MRC)

else if Node Type $==$ Mesh Client then

++ Mesh Client Count(MCC)

end if

End if

Get the Interface Link Quality(ETX)

Calculate the CPLMC values based on Eq. 4

$C P L M C \geq C P L M C_{\text {Max }}$

$C P L M C_{\text {Min }}$ Routing Metric Value

If $C P L M C_{M i n}==$ Many Values

(then Shortest path)

else

Return the $C P L M C_{\text {Min }}$

Select the Optimal Route
Figure 2 NEARMesh Pseudocode

The proposed mesh network scheme (NEARMesh) ensures that only stable nodes with the required power level, link quality and capacity can participate in data transmission. We introduce a Power Level Index (PLI), two different power thresholds (low and high) and ETX, which measures the bidirectional link quality. The scheme also checks the mesh nodes' status. All of these information-aware metrics are sent back to the data sources and are used to calculate the routing metric value. For instance, if the $k$ th mesh Client (MC in Figure 1) wants to send messages to the mesh router (MR) in the $c$ th headquarter, the path from $k$-to- $c$ must meet the quality requirements specified by the NEARMESH scheme, e.g. CPLMC in Figure 2. The abbreviations used in the NEARMesh algorithm Pseudocode are listed in TABLE 1.

\section{Adapted OSPF Protocol}

\section{Standard OSPF}

The widely researched and developed adaptive routing protocol OSPF [10] is employed in this paper. OSPF uses a link state routing algorithm and has two basic mechanisms to determine the link state. The first mechanism focuses on the 
Link State Advertisements ( $L S A S$ ) which are generated by each node, carrying the status of its entire set of links along with their costs. These advertised messages are flooded throughout the network. The second mechanism is Hello Packets $(H L)$, which determine if the link to a given neighbour is still alive by sending a hello packet within the interval window. OSPF draws network map by collecting information. One mechanism that can be used to improve the performance of OSPF is a cost metric. The metric of an interface in OSPF is an indication of the overhead required to send packets across a particular interface. The OSPF cost of an interface is inversely proportional to the bandwidth of that interface $(\operatorname{Cos} t=1 / f)$. So, a higher bandwidth indicates a lower cost.

\section{OSPF_MNTA}

Standard OSPF has no mechanism for establishing routes that preferentially traverse mesh routers. It selects nodes based upon their interface cost (metric) value. However, this metric has no approach to recognize node types. This limitation means mesh clients are frequently used in an end-to-end path, whereas mesh routers have more attractive characteristics for use than mesh clients, due to their stability and increased capacity [11]. As a result, the routes established using standard OSPF are practically of poor quality in WMNs for incident areas. This limitation has been treated by involving a mesh node-type aware (MNTA) routing metric, as follows:

$$
\mathrm{MNTA}=\alpha \times \Sigma(M R C)_{i}+\beta \times \Sigma(M C C)_{j}
$$

The node-aware routing metric consists of two summation segments: mesh router counts (MRC) and mesh client counts (MCC). The parameters $\alpha$ and $\beta$ are the priority weights associated with mesh routers (MR) and mesh clients (MC) along the path, respectively.

\section{OSPF_ICP}

The node power prolongs network connectivity, which is a very important factor in incident area WMNs [6]. Routing protocols have no robust algorithm for establishing routes based upon their node's power level [12]. The Inverse Current Power (ICP) routing metric addresses this problem for use in incident area WMNs. The ICP metric measures the quantity of power in a particular node $I C P_{t+\Delta t}=1 / C P_{t+\Delta t}$ where $C P_{t+\Delta t}$ is the current power for that node. $P_{i}$ and $P_{j}\left(P_{i}>0, P_{j}>0\right)$ are the current normalized power of the next-hop Node $i$ (an MR), or Node $j$ (an $\mathrm{MC}$ ). The current power $(C P)$ for a node is calculated as $C P_{t+\Delta t}=$

$$
C P_{t}-P R x \times S \times n-P T x \times S \times m-P O \operatorname{Pher}(\Delta t),
$$

where $S$ is the size of the packet in bits, and $n$ and $m$ are the number of trials for a successful reception and transmission, respectively. If $C P \leq 0$ (or an alternative nonzero threshold), the node is pre-excluded from the route selection; otherwise, $C P$ is normalized to $P(P \in[0,1])$ and the node is a potential participant. It is noted that radio communication is the dominant power consumer. However, the ICP metric evaluates the power node efficiency for receiving/forwarding packets in such networks; as a result, link quality is ignored.

\section{CPLMC Routing Metric}

Neither reactive nor proactive routing protocols have an integrated mechanism for establishing routes based on joint metrics such as a node's power level [5], link quality and the mesh node type [3]. Most network protocols select nodes based on their respective single routing metric. However, these metrics have no way to consider power level in the next-hop in order to prolong node connectivity. As a consequence, paths established based on these concepts are lack power, have poor link quality, less capacity and stability in incident area WMNs. This work addresses this limitation by introducing the CPLMC routing metric. In the NEARMesh scheme, the Cumulative Power_Link Mesh Cost (CPLMC) is combined with the power node efficiency, link quality and node capacity and stability. The CPLMC metric is cumulative for routing cost, using a specific collection of mesh routers (the first component) and mesh clients (the second component) for any given end-to-end path. This combination represents link quality multiplied by inverse power for both types of mesh (router and clients) along the path. Additionally, the mesh client count (MCC) is added to the mesh client component, as defined below:

$$
\begin{aligned}
C P L M C= & \alpha \times \sum_{i \in \text { path }}\left(\frac{1}{d f \times d r_{i}} \times \frac{1}{C P_{t+\Delta t}}\right)+ \\
& \beta \times \sum_{j \in \text { path }}\left(\frac{1}{d f \times d r_{j}} \times \frac{1}{C P_{t+\Delta t}}+\sum M C C\right) .
\end{aligned}
$$

Then,

$$
\begin{gathered}
C P L M C=\alpha \times \sum_{i \in \text { path }}\left(\operatorname{ETX}_{i} \times \frac{1}{P_{i}}\right)+ \\
\beta \times \sum_{j \in \text { path }}\left(\text { ETX }_{j} \times \frac{1}{P_{j}}+\sum M C C\right) .
\end{gathered}
$$

Parameters $\alpha$ and $\beta$ are the priority weights associated with mesh routers (MR) and mesh clients (MC), respectively, along the path. To prioritize the use of mesh routers wherever feasible, $\alpha$ is set to be several times less than $\beta$, since mesh routers are by far preferable to serve as relay nodes. For demonstration purpose, their values are fixed in this work as $\alpha$ $=1$ and $\beta=4$, although these values are adjustable based on empirical trials. $i$ and $j$ indicate a next-hop MR or MC node along the path, or the link connected to the next-hop node.

$E X T$ is a link quality metric, as defined above by measuring the cost of the output interface's link to the nexthop, which is a very significant factor in incident areas. The parameters $d f$ and $d r$ are the forward and reverse delivery ratios of the link between two nodes $a$ and $b$, respectively. The reverse delivery ratio of a link $d r$ at $a$ is typically calculated using successfully received periodic link probe packets from $b$, 
divided by the expected total number of probe packets from $b$ during a given time window. The forward delivery ratio of the link $d f$ at $a$ is equivalent to the reverse delivery ratio $d r$ at $b$. Therefore, $a$ can derive the ETX for the link by collecting the $d f$ (i.e., the $d r$ calculated at $b$ ) from $b$. Link probe packets (usually called Hello messages) are generally implemented in ad hoc routing protocols. A successful transmission in both directions is essential, with corresponding probability of $d f \times d r$. The CPLMC routing metric values are updated in routing tables. The optimal route to a certain destination is the recent lowest CPLMC value. These values are broadcast by LSAs over the network within the interval window or if instant change in the network topology.

\section{Simulation Results}

\section{Simulation Environment}

The NCTUns simulator [12] is employed in this study to implement and evaluate the Mesh Incident Area Network (MINA) for communications between the emergency management office and a rescue team. The proposed NEARMesh scheme, based on the CPLMC routing metric, is compared with the standard OSPF routing protocol, OSPF_MNTA and OSPF_ICP. The default simulation parameters used in the scenarios are listed in Table 2. The performance measurements were obtained by averaging the results over 20 test runs. We considered the following performance evaluation parameters: quality of service (QoS) metrics, including packet delivery ratio, routing overhead and end-to-end delay, and total consumed energy of the mesh client's nodes during simulation time.

TABLE 2: The Parameter Settings USEd In the Simulations

\begin{tabular}{|l|c|}
\hline \multicolumn{1}{|c|}{ Parameter } & Value \\
\hline Simulated time (s) & 400 \\
\hline Simulation area $\left(\mathrm{m}^{2}\right)$ & $1000 \times 1000$ \\
\hline Wireless link bandwidth (Mbps) & 11 \\
\hline Propagation model & Two-ray ground \\
\hline Mobility model for mesh clients & Random waypoint \\
\hline Minimum packet size (Bytes) & 64 \\
\hline Transmission mode & Full-duplex \\
\hline Packet rate (kbps) & 128 \\
\hline Transmission range (m) & 250 \\
\hline Transmission power (dBm) & 20 \\
\hline Number of mesh clients & 10 \\
\hline Number of mesh routers & 20 \\
\hline Maximum speed of mesh client (m/s) & 25 \\
\hline Initial node power (PL) (w) [13] & 200 \\
\hline Tx, Rx (micro watt) [13] & 1.75 \\
\hline
\end{tabular}

\section{Results and Evaluation}

Scenario 1: Varying the speed of the Mesh_Client

Scenario 1 varied the speed of mesh clients from $0 \mathrm{~m} / \mathrm{s}$ to $25 \mathrm{~m} / \mathrm{s}$. The corresponding performance metrics for OSPF, OSPF_MNTA, OSPF_ICP and NEARMesh, shown in Figure 3 , indicate that, when the mesh clients are static (zero speed), NEARMesh achieved a 95\% Packet Delivery Ratio (PDR), considerably higher than that obtained by the standard OSPF, OSPF_MNTA and OSPF_ICP, of $79 \%, 85 \%$ and $80 \%$, respectively. With speed-up, as mobility introduced topology changes and router reselection, PDR declined. This is attributed to increased packet loss during the transition. For instance, at 5 $\mathrm{m} / \mathrm{s}$, the PDR of standard OSPF, OSPF_ICP and OSPF_MNTA dropped to $40 \%, 60 \%$ and $65 \%$ respectively. At the maximum speed of the mesh client, $25 \mathrm{~m} / \mathrm{s}$, (rescue vehicles) the PDR of standard OSPF, OSPF_ICP and OSPF_MNTA dropped 33\%, $52 \%$ and $50 \%$, respectively. Compared with this, NEARMesh maintained significantly higher PDR at around $80 \%$.

This superior performance was largely attributed to the beneficial combination of both OSPF_MNTA and OSPF_ICP routing metrics in one, called CPLMC. Firstly, OSPF_MNTA preferred utilization of mesh routers, which are static, in route selection, and thus minimized the degree of topology changes and route recovery time and overhead. Secondly, OSPF_ICP is node power- aware metric which allows nodes that possesses excellent amount of power to be involved in the route. Lastly, the link quality has been considered by including ETX, thanks to the integrated CPLMC metrics in NEARMesh. It is further noted that the routes created using standard OSPF, OSPF_MNTA and OSPF_ICP sometimes suffered from significant performance degradation due to dead nodes (nodes running out of battery). However, NEARMesh uses the poweraware routing metric and routes are established through nodes with enough remaining power. The combination of power sufficiency assurance and the prioritized employment of static nodes (i.e., mesh routers) in route selection resulted in more stable routes to facilitate more reliable and efficient, and faster communications. As a result, the routing overhead of NEARMesh is less, compared with OSPF_ICP and OSPF_MNTA, as shown in Figure 4, with increasing the mesh client's speed. For instance, when the speed was around $15 \mathrm{~m} / \mathrm{s}$, the mesh clients moved closer to each other, due to the confined movement area in our study. This waypoint movement leads to an extensive contention area, additionally resulting in a high delay time for others. However, the NEARMesh delay is less than 200s at the client's minimum speed. Furthermore, the delay drops to $100 \mathrm{~ms}$ at client's maximum speed. 


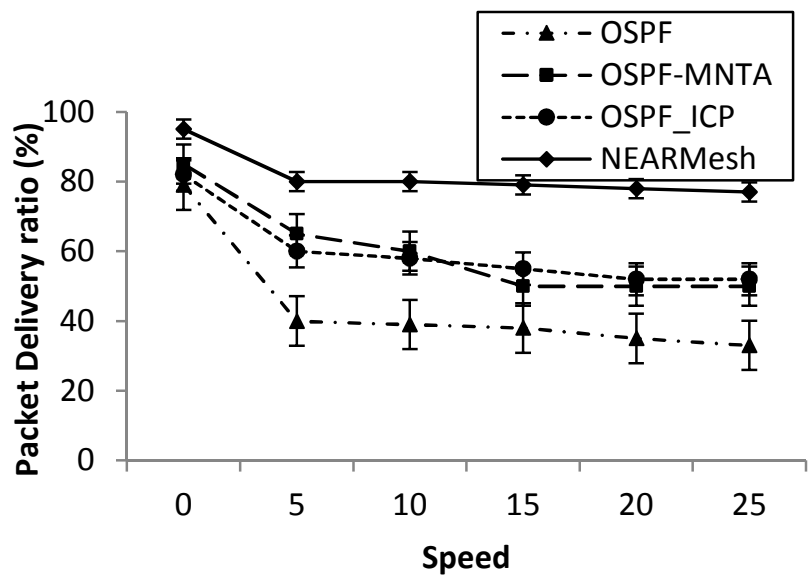

Figure 3: Packet delivery ratio vs. mesh client speed

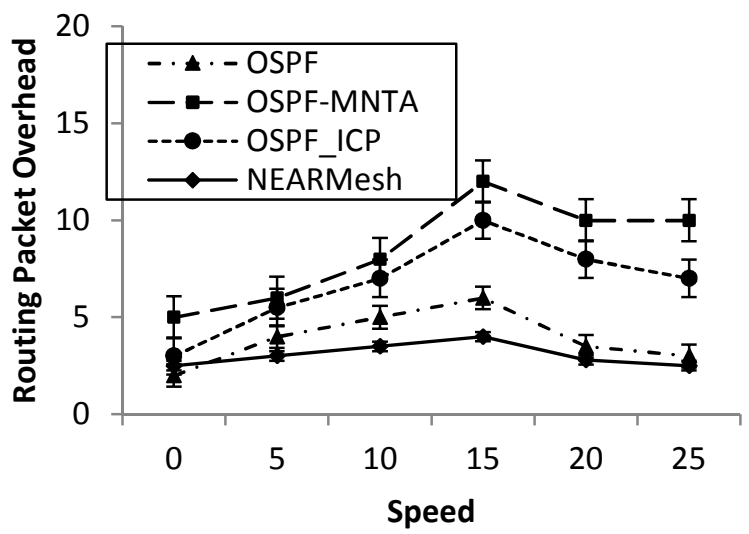

Figure 4: Routing packet overhead vs. mesh client speed

Scenario 2: Varying the number of Mesh_Routers

In order to study the impact of deploying more mesh routers in the backbone network, the number of mesh routers considered in this scenario was $\mathrm{n}=0,4,8,15$, and 20 . Figure 5 shows superior PDR results in NEARMesh, in contrast to OSPF_ICP, standard OSPF and OSPF_MNTA, respectively. The paths' availability is limited when the number of mesh routers is zero. Despite operating the in the same channel, mesh clients have less capacity and stability than the mesh routers which thus increases the contention for the wireless medium, thereby causing frequent route breaks. However, as soon as the number of mesh routers was increased, the PDR of NEARMesh improved more quickly compared with standard OSPF, OSPF_ICP and OSPF_MNTA. This was due to the fact that NEARMesh was able to take better advantage of the growing availability of intermediate mesh routers in route selection, benefiting from both the node power awareness and the bi-directional link quality being measured by including ETX. The joint capabilities of the intermediate mesh routers, node power awareness and link quality alleviate the contention area. As a result, there is more capacity, with stability, prolonging network connectivity, minimising route breaks, with less routing overheads and low delay, as shown in Figure 6 , due to increases in the routes being selected. Thus, when no mesh was deployed in the backbone network the delay for most routing metrics lay between $500 \mathrm{~ms}$ and $700 \mathrm{~ms}$. As soon as the number of mesh routers was increased, the delay dropped to $580 \mathrm{~ms}$ in the case of standard OSPF, 500ms for OSPF_MNTA, $350 \mathrm{~ms}$ for OSPF_ICP, and 150ms for NEARMESH.

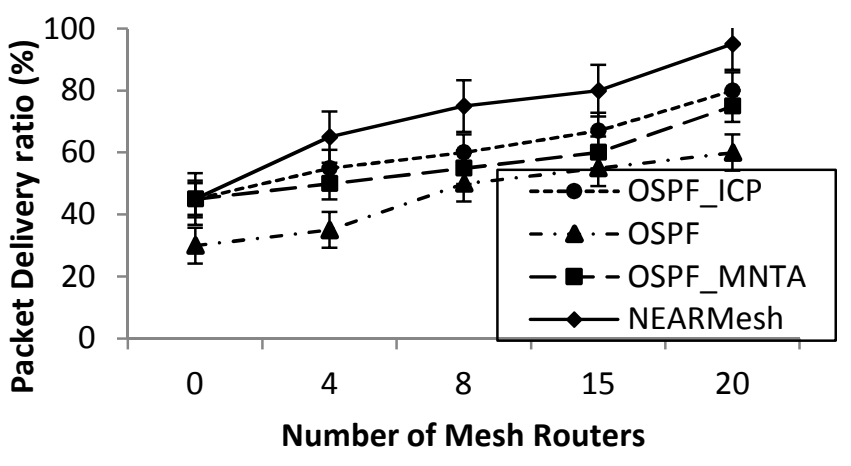

Figure 5: Packet delivery ratio vs. number of mesh routers

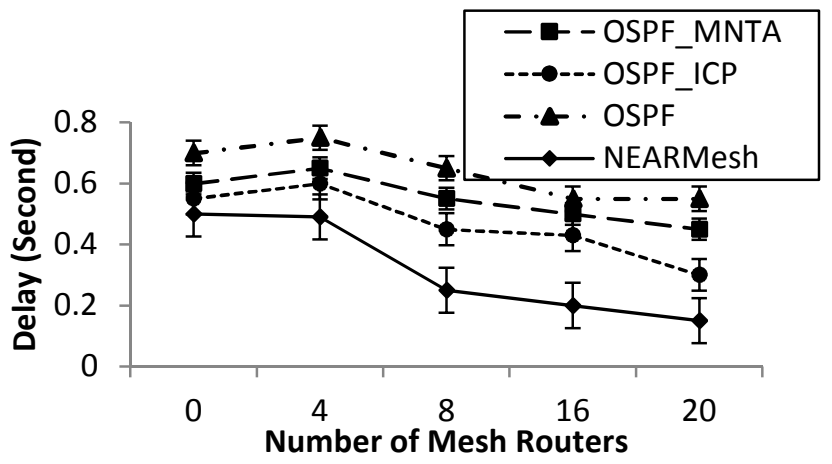

Figure 6: Delay vs. number of mesh routers

\section{Evaluation of NEARMESH Power Consumption}

An energy-efficient platform is imperative during emergency management. The network environment aware routing scheme (NEARMesh) was evaluated by measuring the mesh client's power consumption for all routing schemes. Figure 7 shows the mesh client's average power consumption in NEARMesh, OSPF_ICP, OSPF_MNTA and Standard OSPF. All mesh clients contributed to communication (sending/receiving packets) through the backbone network. Thus, mesh clients consume more energy than static routers. The mesh clients MC4, MC5, MC6, and MC7 consumed more energy in OSPF_ICP, OSPF_MNTA and Standard OSPF, compared with the case in NEARMesh, which consumed less energy. This is attributed to their contribution to the network and wise regulation of energy in the NEARMesh case. However, a smaller amount of energy was consumed by mesh clients MC1, MC2, MC3, MC8 MC9 and MC10. The average 
power consumed in NEARMesh, OSPF_ICP, OSPF_MNTA and Standard OSPF routing schemes was $26 \%, 38 \%, 47 \%$ and $60 \%$ respectively. Thus, notable power conservation observed in the NEARMesh scheme, mainly due to the fact that NEARMesh generated fewest control packets to establish the route, and employed the integrated alertness routing metrics which are power, mesh node type aware and link quality. The NEARMesh, OSPF_ICP, OSPF_MNTA and Standard OSPF routing schemes were able to save power up to an average of $74 \%, 62 \%, 53 \%$ and $40 \%$, respectively.

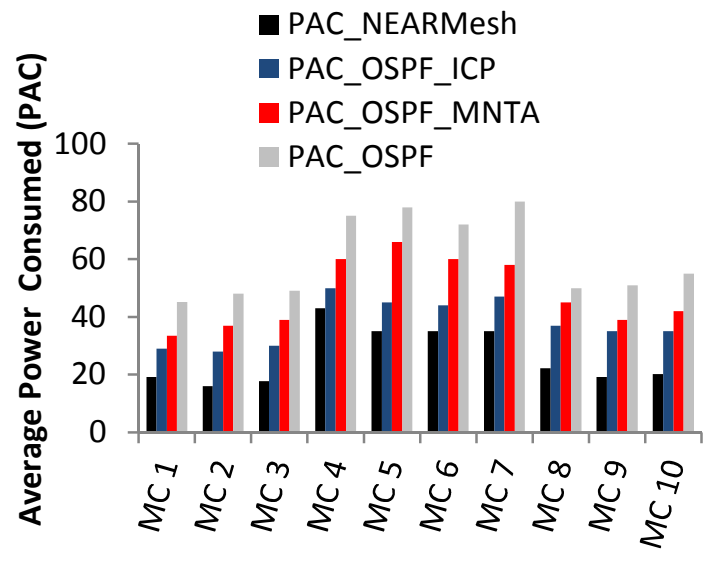

Mesh Client Node

Figure 7: Energy consumption for mesh clients using each scheme

\section{Conclusion}

One major challenge in emergency response network communications lies in providing reliable and efficient routes. Emergency environment conditions are different from other kind of network communications. This article has proposed a network-environment-aware routing (NEARMesh) scheme for WMNs which employs the power level, mesh node type and link quality awareness to find the optimal route to the final destination. This scheme aimed to prolong network lifetime connectivity, forward packets through high capacity nodes and ensure link quality. The simulation results demonstrate that NEARMesh significantly outperformed the standard OSPF, OSPF_MNTA and OSPF_ICP. In all scenarios, NEARMesh managed to achieve highest packet deliver ratio and lowest end-to-end delay, whilst considerably reducing the routing overhead and total power consumption.

Acknowledgments: This work is supported by the Ministry of Higher Education (MOHE) and Research Management Centre (RMC) at the Universiti Teknologi Malaysia (UTM) under the Research University Grant Category (VOT Q.J130000.2528.16H74).

\section{References}

[1] Z. Chen, L. Chen, Y. Liu, and Y. Piao, "Application Research of Wireless Mesh Network on Earthquake", Proc. IEEE International Conference on Industrial and Information Systems, Haikou, China, pp. 19-22, 2009.

[2] G. Calarco and M. Casoni, "Virtual Networks and Software Router approach for Wireless Emergency Networks Design”, Proc. IEEE $73^{\text {rd }}$ Vehicular Technology conference, Budapest, May 2011.

[3] A. Pirzada, M. Portmann, R. Wishart, and J. Indulska, "SafeMesh: A Wireless Mesh Network Routing Protocol for Incident Area Communications", Pervasive and Mobile Computing, Vol. 5, pp. 201-22, 2009.

[4] T.A. Hadhrami, Q. Wang, and C. Grecos, "Real-Time Visual Communication to Aid Disaster Recovery in a MultiSegment Hybrid Wireless Networking System," Real-Time Image and Video Processing, SPIE, vol. 8437, pp. 1-14, 2012.

[5] P. Pathak and R. Dutta, "A Survey of Network Design Problems and Joint Design Approaches in Wireless Mesh Networks," Communications, vol. 13, 2011, pp. 396-428.

[6] A. Akhtar, M. Nakhai, and A. Aghvami, "Adaptive PowerAware Routing in Wireless Mesh Networks," Energy, 2011.

[7] A. Akhtar, M. Nakhai, S. Member, and A. Aghvami, "Power Aware Cooperative Routing in Wireless Mesh Networks," Communications, vol. 16, pp. 670-673, 2012.

[8] I. Stojmenovic and X. Lin, "Power-aware localized routing in wireless networks," 2001, pp. 1-22.

[9] A. Raniwala, "Centralized Channel Assignment and Routing Algorithms for Multi-Channel Wireless Mesh Networks," Mobile Computing and Communications Review, vol. 8.No.2, 2004.

[10] A. Roy and M. Chandra, "Extensions to OSPF to support mobile ad hoc networking. Internet-Draft, draft-ietf-ospfmanet-or00, Work in Progress, February 2008.

[11] A. Pirzada and M. Portmann, High Performance AODV Routing Protocol for Hybrid Wireless Mesh Networks, in Proceedings of the 4th Annual International Conference on Mobile and Ubiquitous Systems: Computing, Computing Networking and Services MOBIQUITOUS, 2007

[12] S. Wang, and C. Chou, "NCTUns Tool for Wireless Vehicular Communication Network Researches," Simulation Modelling Practice and Theory, Vol. 17, pp. 1211-1226, 2009

[13] NS, The Network Simulator, 1989, http://www.isi.edu/nsnam/ns/.

[14] T.A. Hadhrami, Q. Wang, and C. Grecos, "Power-and Node-Type-Aware Routing Algorithm for EmergencyResponse Wireless Mesh Networks", IEEE $77^{\text {th }}$ Vehicle Technology Conference (VTC-Spring 2013), Dresden 2013. 